\title{
Resultado de la aplicación del protocolo de manejo de pacientes con cáncer de ovario en el Hospital Universitario del Valle en el período Enero 94 - Diciembre 97
}

\author{
José Saulo Torres*; Juan Pablo Suso**; Eduardo Perea**; Luis Alberto Tafur***; Luis Fernando Tobón ${ }^{* * * *}$
}

\section{RESUMEN}

Para asegurar el cumplimiento de los protocolos establecidos en el manejo de las pacientes oncológicas es indispensable una conciencia colectiva de apoyo de todos los estamentos que directa o indirectamente rodean al paciente; permitiendo que se cumplan cada uno de los objetivos indispensables para poder llegar a un beneficio común el cual no es otro que la calidad de vida del paciente.

PALABRAS CLAVES: Cáncer de ovario, paciente, equipo médico, instituciones gubernamentales y no gubernamentales.

\section{SUMMARY}

To ensure compliance with established protocols for treating oncological patients, it is essential to be collectively supportive and aware of all the factors that either directly or indirectly affect the patients; making it possible toachieve each indispensable objective and obtain a common benefit, which is the patient's quality of life.

KEY WORDS: Ovary Cancer, Patient, Medical Team, Governmental and Non-Governmental Organizations.

\section{INTRODUCCION}

El carcinoma de ovario es la malignidad más letal del aparato genital femenino. El $76 \%$ de los pacientes se detectan en estadios III y IV(1). La supervivencia se relaciona directamente con el estadio clínico y el tratamiento. La piedra angular del tratamiento es la cirugía, la cual permite un diagnóstico adecuado, estadificación, y citorreducción óptima; y es la base para la escogencia de un tratamiento complementario (2).

La cirugía clasificatoria consiste en una laparotomia mediana infra-supraumbilical, citologias de goteras paietocolicas, y cúpulas diafragmaticas y tumorectomia. Una vez se confirme la malignidad por la biopsia por congelación se procede a realizar omentectomía, muestreo ganglionar pélvico del lado del tumor, muestreo ganglionar paraortico y apendicectomía. Un esfuerzo máximo en la citorreducción es imperativo, puesto que residuos tumurales mínimos están asociados con mejor sobrevida (3)

La laparotomía de segunda mirada se efectúa en : Pacientes que se consideren clínica y paraclinica libres de enfermedad; se les pueda ofrecer una segunda línea de

\footnotetext{
* Jefe sección Ginecología Oncológica H.U.V. docente Ad-honorem Universidad del Valle Cali Colombia

Ginecólogos Oncólogos H.U.V. Docentes Ad-honorem Universidad del Valle

Especialista Salud Pública. Profesor Titular Escuela de Salud Pública Universidad del Valle

Ginecólogo, Médico Hospitalario H.U.V.
}

tratamiento y se acepte el procedimiento por parte del paciente (4).

Cuando la quimioterapia inicial ha sido a base de cisplatino se recomienda en tumores epiteliales practicar la laparotomía de segunda mirada después de los siguientes seis meses (5).

Para tumores germinales sin marcador tumoral positivo, se recomienda realizar segunda mirada precozmente al terminar la quimioterapia, la cual determina la continuidad o no de los quimioterapicos(6).

Nuestro protocolo de qumioterapia para tumores epiteliales es a base de Ciclofosfamida - Cisplatino y en segunda instancia Carboplatino Ciclofosfamida. La quimioterapia para tumores epiteliales se recomienda a partir del estadio clínico IC o en Estadios IA o IB con grados histologicos 3(7).

La decisión de administrar la quimioterapia se toma en junta multidisciplinaria, entre 10 y 15 días después de la cirugía, y se administra en forma ambulatoria en la sección de quimioterapia. Una vez la paciente termina todos los ciclos es reintegrada al servicio de Oncología Ginecológica continuando los controles por los dos servicios de acuerdo con las normas del protocolo.

La radioterapia se recomienda en pacientes con cirugía óptima y que por dificultad económica no tengan acceso a la quimioterapia, o en pacientes que a la laparatomia de segunda mirada, tengan enfermedad residual mínima y por razones similares no puedan acceder a una quimioterapia de segunda línea.

En este artículo presentamos un análisis del resultado de este manejo. 


\section{OBJETIVOS}

Analizar el resultado de la aplicación del protocolo de manejo de las pacientes con diagnóstico de cáncer de ovario atendidas entre Enero de 1994 y Diciembre de 1997 en el Hospital Universitario del Valle.

\section{MATERIALES Y METODOS}

Se realizó una revisión retrospectiva de las historias de las pacientes que ingresaron al Hospital Universitario del Valle de Cali - Colombia con diagnóstico de tumor de ovario. De las 199 pacientes admitidas en el período comprendido de enero de 1994 a diciembre de 1997, se estudiaron 77 pacientes con diagnóstico de cáncer de ovario.

Teniendo en cuenta que las pacientes operadas en la institución se les diligencia un formulario consignando el resultado de la cirugía y el volumen tumoral. (Ver Anexo 1), se tabuló el formulario para la obtención de los datos.

El estado de la paciente a Diciembre de 1997 (viva o muerta) se obtuvo a través de llamadas telefónicas.

\section{RESULTADOS}

Se diagnosticarón 77 tumores primarios y cuatro metastasicos, el diagnóstico final se especifica en la tabla I

En la tabla 2 se presenta la distribución por edades según tipo histológico

El $43 \%$ de los tumores de ovario corresponden a estadios tempranos, (E I) y el $57 \%$ a estadios avanzados (E II, III y IV) (tabla 3)

Tabla 1

DISTRIBUCION POR GRUPOS Y TIPOS HISTOLOGICOS. H.U.V.

\begin{tabular}{|l|c|}
\hline \multicolumn{1}{|c|}{ TIPOS HISTOLOGICOS } & Total \\
\hline EPITELIALES & $\mathbf{6 2}$ \\
\hline Carcinoma seroso papilar & 44 \\
\hline Carcinoma Mucinoso & 11 \\
\hline Carcinoma endometrial & 6 \\
\hline Carcinoma células claras & 1 \\
\hline GERMINALES & $\mathbf{5}$ \\
\hline Disgerminoma & 2 \\
\hline Teratoma inmaduro & 2 \\
\hline Carcinoma Embrionario & 1 \\
\hline ESTROMALES & $\mathbf{9}$ \\
\hline Tumor de células de la granulosa & 8 \\
\hline Tumor de células de la granulosa juvenil & 1 \\
\hline METASTÁSICOS & $\mathbf{4}$ \\
\hline Metastásicos & 4 \\
\hline SARCOMA & $\mathbf{1}$ \\
\hline sarcoma & 1 \\
\hline Total & $\mathbf{8 1}$ \\
\hline
\end{tabular}

Tabla 2

DISTRIBUCION POR EDAD VS TIPOS HISTOLOGICOS. H.U.V.

\begin{tabular}{|c|c|c|c|c|c|c|}
\hline EDAD & $\begin{array}{c}\text { EPITE- } \\
\text { LIAL } \\
77 \%\end{array}$ & $\begin{array}{c}\text { ESTRO- } \\
\text { MALES } \\
11 \%\end{array}$ & $\begin{array}{c}\text { GERMI- } \\
\text { NALES } \\
6 \%\end{array}$ & $\begin{array}{c}\text { METAS- } \\
\text { TASICOS } \\
5 \%\end{array}$ & $\begin{array}{c}\text { SARCO- } \\
\text { MAS } \\
1 \%\end{array}$ & TOTAL \\
\hline $10-15$ & 1 & 1 & & & & 2 \\
\hline $16-20$ & & & 3 & & & 3 \\
\hline $21-30$ & 3 & 1 & 2 & & & 6 \\
\hline $31-40$ & 8 & 3 & & & & 11 \\
\hline $41-50$ & 23 & 2 & & 3 & 1 & 29 \\
\hline $51-60$ & 16 & 1 & & & 1 & 17 \\
\hline$>60$ & 11 & 1 & & 1 & & 13 \\
\hline Total & 62 & 9 & 5 & 4 & 1 & 81 \\
\hline
\end{tabular}

Tabla 3

TUMORES DE OVARIO. ESTADIO CLINICO VS. GRUPO HISTOLOGICO .H.U.V.

\begin{tabular}{|c|c|c|c|c|c|c|}
\hline ESTADIOS & $\begin{array}{c}\text { EPITE- } \\
\text { LIAL }\end{array}$ & $\begin{array}{c}\text { ESTRO- } \\
\text { MALES }\end{array}$ & $\begin{array}{c}\text { GERMI- } \\
\text { NALES }\end{array}$ & $\begin{array}{c}\text { METAS- } \\
\text { TASICO } \\
\text { S }\end{array}$ & $\begin{array}{c}\text { SARCO- } \\
\text { MAS }\end{array}$ & Total \\
\hline IA & 16 & 8 & 4 & & & 28 \\
\hline IC & 7 & & & & & 7 \\
\hline IIC & 2 & & & & & 2 \\
\hline IIIA & 3 & & & & & 3 \\
\hline IIIB & 11 & & & 1 & & 12 \\
\hline IIIC & 16 & & 1 & & 1 & 18 \\
\hline IV & 1 & 1 & & & & 2 \\
\hline IVA & 1 & & & 1 & & 2 \\
\hline IVB & 4 & & & & & 4 \\
\hline No Aplica & & & & 2 & & 2 \\
\hline No clasific & 1 & & & & & 1 \\
\hline Total & 62 & 9 & 5 & 4 & 1 & 81 \\
\hline
\end{tabular}

El Grado Histológico 3 tuvo más mortalidad. De los 8 tumores de B.P.M. siete fueron IA, y uno IC ninguna recibió quimioterapia y todas se encuentran vivas y libres de enfermedad. Ver tabla 4.

En las tablas siguientes se excluyen los tumores metastasicos.

Tabla 4

GRADO DE DIFERENCIACIÓN HISTOLÓGICO T EPITELIALES H.U.V.

\begin{tabular}{|c|c|c|c|c|c|}
\hline Grado H & B.P.M & $\mathbf{G}^{\mathbf{0}}$ & $\mathbf{G}^{\mathbf{0}} \mathbf{2}$ & $\mathbf{G}^{\mathbf{3}}$ & total \\
\hline Sobrevive & 8 & 12 & 10 & 9 & 39 \\
\hline Murió & & 3 & 3 & 4 & 10 \\
\hline Se perdió & & 1 & 7 & 5 & 13 \\
\hline Total & 8 & 16 & 20 & 18 & 62 \\
\hline
\end{tabular}

Tabla 5

TIPO HISTOLÖGICO VS SOBREVIDA. H.U.V.

\begin{tabular}{|c|c|c|c|c|c|}
\hline & Epitelial & Estromal & Germinal & Sarcomas & total \\
\hline vive & 39 & 6 & 4 & 0 & 49 \\
\hline Murió & 10 & 0 & 0 & 0 & 10 \\
\hline Se Perdió & 13 & 3 & 1 & 1 & 18 \\
\hline Total & 62 & 9 & 5 & 1 & 77 \\
\hline
\end{tabular}


Del grupo de pacientes con Cáncer de ovario 49 viven, 10 murieron y de 18 no se obtuvo ninguna información. Ver Tabla 5.

De las 77 pacientes en quienes se practicó una cirugía clasificatoria la citorreducción se considero óptima en 62 $(81 \%)$. Ver tabla 6.

De las 46 pacientes que fueron seleccionadas para recibir quimioterapia, 27 (59\%) la recibieron en forma adecuada, de estas solo una murió.

Tres pacientes que no tenían acceso económico a la qumioterapia se remitieron a radioterapia, Ver tabla 7.

La tabla 8 muestra los diez casos de muerte durante este período.

\section{DISCUSION:}

De las 77 pacientes con tumores malignos de ovario operadas en este período 49 están vivas, 18 se perdieron y

\section{Tabla 6}

ESTADO DE LAS PACIENTES A QUIENES SE LES PRACTICO CIRUGÍA CITORREDUCTORA. H.U.V.

\begin{tabular}{|l|c|c|c|c|c|}
\hline & $<\mathbf{2 c m}$ & $>\mathbf{2 c m}$ & Inoperables & Sin datos & Total \\
\hline Sobrevive & 44 & 5 & 0 & 0 & 49 \\
\hline Murió & 6 & 2 & 1 & 1 & 10 \\
\hline Se Perdió & 12 & 2 & 1 & 3 & 18 \\
\hline Total & 62 & 9 & 2 & 4 & 77 \\
\hline
\end{tabular}

Tabla 7

QUIMIOTERAPIA VS. SOBREVIDA

\begin{tabular}{|l|c|c|c|c|}
\hline Tratamiento Complementario & Vive & Murió & Se perdió & Total \\
\hline Quimioterapia completa & 23 & 1 & 3 & 27 \\
\hline Quimioterapia Incompleta & 0 & 3 & 4 & 7 \\
\hline Quimioterapia ordenada y no recibida & 1 & 6 & 5 & 12 \\
\hline Quimioterapia no necesitaba & 24 & 0 & 4 & 28 \\
\hline Radioterapia & 1 & 0 & 2 & 3 \\
\hline Total & 49 & 10 & 18 & 77 \\
\hline
\end{tabular}

Tabla 8

CASOS DE MUERTE DE CA DE OVARIO CONFIRMADOS.H.U.V.

\begin{tabular}{|c|c|c|c|c|}
\hline tipo & Estadio & EDAD & CIRUGIA & QUIMIOTERAPIA \\
\hline E & IIIC & 56 & $<2 \mathrm{~cm}$ & C \\
\hline E & IIIC & 55 & $<2 \mathrm{~cm}$ & I $^{*}$ \\
\hline E & IIIC & 56 & I & NN \\
\hline E & IIIC & 58 & $<2 \mathrm{~cm}$ & $I^{*}$ \\
\hline E & IIIC & 47 & $<2 \mathrm{~cm}$ & I $^{*}$ \\
\hline E & IIIB & 66 & $>2 \mathrm{~cm}$ & NN \\
\hline E & IVB & 49 & $<2 \mathrm{~cm}$ & NN \\
\hline E & IV & 62 & I & NN \\
\hline E & IIIC & 47 & $<2 \mathrm{~cm}$ & NN \\
\hline E & IVA & 49 & $>2 \mathrm{~cm}$ & NN \\
\hline
\end{tabular}

\section{C: Completa}

NN: Necesitó y no la recibió

I: Incompleta

I* Inoperable. diez fallecieron. Se seleccionaron 47 para quimioterapia, de éstas 24 están vivas, 10 murieron y 12 se perdieron.

Todas las que fallecieron fueron programadas para recibir quimioterapia, ninguna la recibió. Solo a una se le administró en forma completa.

Sobrevivientes: En cuanto a los 49 sobrevivientes, 39 son epiteliales, de los cuales 16 son estadios IA, 6 estadios IC y el resto estadios II, III y IV. Tablas IX y X.

La quimioterapia no se recomendó en ninguno de los estadios IA Grado 102.

Los 17 Epiteliales restantes corresponden a estadios II - IV, de los cuales 12 tienen cirugías óptimas y todos recibieron la quimioterapia en forma adecuada.

Del grupo de los Estromales 6 están vivos y libres de enfermedad. Todos corresponden a estadios IA, ninguno recibió quimioterapia por indicación médica.Tabla XI.

Del grupo de Germinales, 3 corresponden a estadios IA y uno a estadio IIIC, a los cuatro se les practicó cirugía óptima y se administro la quimioterapia tal como se recomendó.

En el análisis de este último grupo de pacientes que sobreviven el denominador común es una cirugía óptima con una adecuada quimioterapia, estos dos elementos que deben de venir siempre de la mano es garantía para una adecuada calidad y cantidad de vida.Tabla XII.

Tabla 9

PACIENTES VIVAS DE CANCER DE OVARIO EPITELIAL ESTADIO I.

\begin{tabular}{|c|c|c|c|c|c|}
\hline Tipo & Estadio & Grupo edad & $\begin{array}{c}\text { No. por } \\
\text { grupo }\end{array}$ & CIRUGIA & $\begin{array}{c}\text { QUIMIO- } \\
\text { TERAPIA }\end{array}$ \\
\hline E & IA & $10-20$ & 1 & $<2 \mathrm{~cm}$ & $\mathrm{~N}$ \\
\hline E & IA & $31-40$ & 4 & $<2 \mathrm{~cm}$ & $\mathrm{~N}$ \\
\hline E & IA & $41-50$ & 5 & $<2 \mathrm{~cm}$ & $\mathrm{~N}$ \\
\hline E & IA & $41-50$ & 1 & $\mathrm{~N}$ & $\mathrm{~N}$ \\
\hline E & IA & $51-60$ & 2 & $<2 \mathrm{~cm}$ & $\mathrm{~N}$ \\
\hline E & IA & Mayor de 60 & 3 & $<2 \mathrm{~cm}$ & $\mathrm{~N}$ \\
\hline E & IC & $21-30$ & 1 & $<2 \mathrm{~cm}$ & $\mathrm{C}$ \\
\hline E & IC & $41-50$ & 2 & $\mathrm{~N}$ & $\mathrm{C}$ \\
\hline E & IC & $51-60$ & 1 & $<2 \mathrm{~cm}$ & $\mathrm{C}$ \\
\hline E & IC & $>60$ & 2 & $<2 \mathrm{~cm}$ & $\mathrm{C}$ \\
\hline
\end{tabular}

N: No necesita C: Completa NN: Necesitó y no la recibió

Tabla 10

PACIENTES VIVAS DE CA DE OVARIO EPITELIALES E II-IV

\begin{tabular}{|c|c|c|c|c|c|}
\hline tipo & Estadio & Grupo edad & $\begin{array}{l}\begin{array}{c}\text { No. Por } \\
\text { grupo }\end{array} \\
\text { grupen }\end{array}$ & Cirugía & Quimioterapia \\
\hline $\mathrm{E}$ & IIC & 41 a 50 & 2 & $<2 \mathrm{~cm}$ & $\mathrm{C}$ \\
\hline E & IIIA & 51 a 60 & 1 & $<2 \mathrm{~cm}$ & $\mathrm{C}$ \\
\hline E & IIIB & 41 a 50 & 5 & $<2 \mathrm{~cm}$ & $\mathrm{C}$ \\
\hline $\mathrm{E}$ & IIIC & 31 a 40 & 1. & $<2 \mathrm{~cm}$ & $\mathrm{C}$ \\
\hline $\mathrm{E}$ & IIIC & 41 a 50 & 1 & $<2 \mathrm{~cm}$ & $\mathrm{C}$ \\
\hline$E$ & IIIC & 41 a 50 & 1 & .2 .07 & $\mathrm{C}$ \\
\hline E & IIIC & 51 a 60 & 2 & $<2 \mathrm{~cm}$ & $\mathrm{C}$ \\
\hline $\mathrm{E}$ & IIIC & $>60$ & 2 & / 2 c1n & $\mathrm{C}$ \\
\hline $\mathrm{E}$ & IVB & $51-60$ & 1 & 2.2 .011 & $\mathrm{C}$ \\
\hline $\mathrm{E}$ & IVB & $51-60$ & 1 & - 2 cm & $\mathrm{C}$ \\
\hline
\end{tabular}


Tabla 11

PACIENTES VIVAS DE CA DE OVARIO DEL GRUPO ESTROMA Y CORDONES SEXUALES

\begin{tabular}{|c|c|c|c|c|c|}
\hline tipo & Estadio & $\begin{array}{c}\text { GRUPO DE } \\
\text { EDAD }\end{array}$ & $\begin{array}{c}\text { No. por } \\
\text { grupo }\end{array}$ & Qx & Qt \\
\hline ES & IA & 41 a 50 & 2 & $<2 \mathrm{~cm}$ & N \\
\hline ES & IA & 21 a 30 & 1 & $<2 \mathrm{~cm}$ & N \\
\hline ES & IA & 10 a 20 & 1 & $<2 \mathrm{~cm}$ & N \\
\hline ES & IA & 51 a 60 & 1 & $<2 \mathrm{~cm}$ & N \\
\hline ES & IA & 31 a 40 & 1 & $<2 \mathrm{~cm}$ & N \\
\hline
\end{tabular}

Tabla 12

PACIENTES VIVAS DE CA DE OVARIO TUMORES GERMÍNALES

\begin{tabular}{|c|c|c|c|c|c|}
\hline tipo & Estadio & $\begin{array}{c}\text { Grupo de } \\
\text { edad }\end{array}$ & $\begin{array}{c}\text { No. por } \\
\text { grupo }\end{array}$ & Qx & Qt \\
\hline G & IA & 21 a 30 & 1 & $<2 \mathrm{~cm}$ & N \\
\hline G & IA & 10 a 19 & 2 & $<2 \mathrm{~cm}$ & C \\
\hline G & IIIC & 10 a 19 & 1 & $<2 \mathrm{~cm}$ & C \\
\hline
\end{tabular}

Contrasta esta sobrevida con los pacientes "perdidos" o fallecidas teniendo ambos en común la administración inadecuada de la quimioterapia.

Es importante entrar a analizar las situaciones contribuyentes a que en un porcentaje importante de pacientes no se tuviera acceso a la administración del tratamiento complementario con quimioterapia o se dejara parcialmente iniciado y posteriormente abandonado:

Los motivos por el cual un paciente abandona la terapia complementaria radica en factores dependientes de:

1. El paciente y el entorno familiar.

2. El Equipo Médico

3. Las Instituciones.

\section{Factores del paciente}

- Incapacidad económica.

- Falta de apoyo y Comprensión de la Familia

- Idiosincrasia del paciente y familia.

- Temores

- Información Inadecuada.

- Depresión.

\section{Factores del Equipo Médico}

- Una inadecuada información al paciente.

- La falta de capacidad de poder transmitir en el paciente la confianza necesaria para que no abandone el tratamiento.

- Una infraestructura pobre que no permita la multidisciplinaridad de los pacientes.

\section{Factores de las Instituciones:}

- Garantizarle al paciente no solo lo referente a la estadía hospitalaria para la cirugía sino el compromiso de garantizar la administración de los medicamentos que necesite para su mejoría. Para esto es necesario una sección fortalecida de Trabajo Social.

- Financiación, personalizando la forma de pago de acuerdo a la capacidad económica del paciente.

- Tener un adecuado control por parte de trabajo social, evitando perdidas y deserciones de pacientes.

- Tener Infraestructuras óptimas que soporten los servicios que se ofrecen a los usuarios con responsabilidad, dedicación y calidad.

- Actualizar permanentemente los grupos humanos encargados de orientar en el manejo de las pacientes desde el nivel mas elemental de la atención hasta el mas complejo.

\section{CONCLUSIONES}

1. El paciente con cáncer de Ovario requiere atención integral óptima con grupos bien organizados y consolidados dentro de la institución que permita tener servicios fortalecidos en: Trabajo social, Oncología Ginecologica, psiquiatría, Quimioterapia y Radioterapia

2. Estos grupos Multidisciplinarios deben en forma responsable mantener un seguimiento individual y permanente de cada uno de los pacientes que acudan a su Unidad.

3. El hecho que una paciente reciba en forma adecuada la quimioterapia le garantiza una mejor sobrevida.

4. Se debe recordar que la paciente con cáncer de Ovario tiene tres oportunidades que definen su futuro:

- Una Optima cirugía citorreductora.

- Una óptima quimioterapia y.

- Un seguimiento adecuado.

Estas tres oportunidades no las debe perder el paciente por culpa de grupos e Instituciones responsables de velar por su salud donde ha depositado no solo su confianza sino su futuro.

\section{BIBLIOGRAFIA}

1. Faridullah Q, William P. M, The treatment of Epitelial Ovarian Cancer. Cancer J Clin. 1995; 45.88-101

2. Hoskins WJ. Surgical Staging and Cytoreductive Surgery of Epithelial Ovarian Cancer. 1993; 71,1534-40.

3. NIH Consensus Statement. Ovarian Cancer Screening, Treatment, and Follwup.1994;12(3):1-30

4. van der Burg,MEL More than 20 years second-look surgery in advanced epithelial ovrian cancer: What did we learn?. Anals of Oncology 1997;8: 627-629
5. Podratz KC, Kinney WK. Second-Look Operation in Ovarian Cancer. 1993, 71,1551-8

6. Gershenson DM. Update on Malignant Ovarian Germ Cell Tumors. Cancer. 1993; 71, 1581-90

7. Faridullah Q, McGuire WP, The treatment of Epithelial Ovarian $\mathrm{Ca}_{\text {a }}$ Cancer J Clin. 1995; 45:88-101.

8. Instituto Nazionale Tumori, Milán, Italia. Protocolos y guías de conducta terapéutica. Nuevas tendencias en Oncología. Abril 1993. Volumen $2 \mathrm{~N}^{\circ} 1$ Pag: $26-27$ 
HOSPITAL UNIVERSITARIO DEL VALLE.

SECCIÓN ONCOLOGÍA GINECOLÓGICA.

FECHA :

( ) CIRUGÍA PRIMARIA. （）CIRUGÍA RECLASIFICATORIA. （）SECOND LOOK.

DIAGNOSTICO PRE-OPERATORIO:

DIAGNOSTICO POST-OPERATORIO:

\section{REPERTORIO}

APÉNDICE

BAZO.

CITOLOGÍA DIAFRAG. DER.

CITOLOGÍA DIAFRAG. IZQ.

CITOLOGÍA FONDO DE SACO.

CITOLOGÍA GOTERA P-DER.

CITOLOGÍA GOTERA P-IZQ.

CUELLO UTERINO.

CUERPO UTERINO.

GANGLIOS ILIACOS COMUNES DER.

GANGLIOS ILIACOS COMUNES IZQ.

GANGLIOS ILIACOS EXTERNOS DER.

GANGLIOS ILIACOS EXTERNOS IZQ.

GANGLIOS ILIACOS INTERNOS DER

GANGLIOS ILIACOS INTERNOS IZQ.

GANGLIOS MESENTERICOS.

GANGLIOS OBTURADORES DER.

GANGLIOS OBTURADORES IZQ.

GANGLIOS PARA-ORTICOS

HÍGADO

INFUNDÍBULO OVÁRICO DER.

INFUNDÍBULO OVÁRICO IZQ.

INTESTINO DELGADO.

INTESTINO GRUESO.

LIGAMENTO ÚTERO SACRO DER

LIGAMENTO ÚTERO SACRO IZQ.

LIQUIDO ASCITICO.

MESENTERIO.

OMENTO INFRACOLICO

OMENTO SUPRACOLICO.

OVARIO DERECHO.

OVARIO IZQUIERDO.

PARAMETRIO DERECHO.

PARED ABDOMINAL.

PARAMETRIO IZQUIERDO.

PERITONEO ABDOMINAL PARIETAL. PERITONEO PÉLVICO PARIETAL.

TROMPA DERECHA.

TROMPA IZQUIERDA.

VEJIGA.

* CÓDIGO: 1.=NO, 2.=SI Total, 3.=SI Parcial. 4.= Biopsia

** CÓDIGO: 1.= Negativo. 2.= Positivo

$* * *$ CÓDIGO: $1 .=$ NO. 2.= SI.

CIRUGÍA OPTIMA (VOLÚMENES TUMORALES MENORES DE $2 \mathrm{Cm}$ ) : SI. CIRUJANO:

AYUDANTES:
MUESTREO DE

HISTOLOGIA**

RESIDUO T.***

( )

( )

( )

( )

( )

( )

( )

( )

( )

( )

( )

( )

( )

( )

( )

( )

( )

( )

( )

( )

( )

( )

( )

( )

( )

( )

( )

( )

( )

( )

( )

( )

( )

( )

( )

( )

( )

( )
( )

( )

( )

( )

( )

( )

( )

( )

()

( )

( )

( )

( )

( )

()

( )

( )

( )

( )

( )

( )

( )

( )

( )

( )

( )

( )

( )

( )

( )

( )

( )

( )

( )

( )

( )

(.)

( )
( )

( )

( )

( )

( )

( )

( )

( )

()

( )

( )

( )

( )

( )

( )

( )

( )

( )

( )

( )

( )

( )

( )

( )

( )

( )

( )

( )

( )

( )

( )

( )

( )

( )

( )

( )

( )

( )

( ) 